\title{
PROTECTION CONTRE LES GLACES DES PRISES D'EAU DE LA CENTRALE THERMIQUE DE NANTES-CHEVIRÉ
}

Étude sur modèle réduit

par R. BONNEFILLE, P. GERMAIN ef A. BICHON

Ingénieurs au Laboratoire National d'Hydraulique, Chatou

\section{1. - Généralités}

\subsection{Caractéristiques des ouvrages de prise.}

La centrale thermique de Nantes-Cheviré est située sur la rive gauche de la Loire à quelques kilomètres en aval de Nantes en face de Chantenay (planche 1).

La mise en service s'est effectuée en trois étapes successives, échelonnées de 1953 à 1958 et correspondant à la réalisation de quatre tranches d'équipement :

- tranches 1 et 2 :

2 groupes de $55 \mathrm{MW}$, débit de prise : $6 \mathrm{~m}^{3 /} / \mathrm{s}$;

- tranche 3:

1 groupe de $110 \mathrm{MW}$, débit de prise: $6 \mathrm{~m}^{3 / \mathrm{s}}$;

- tranche 4:

1 groupe de $125 \mathrm{MW}$, débit de prise : $7 \mathrm{~m}^{3 / \mathrm{s}}$.

Les trois ouvrages de prise correspondants, identiques et composés chacun d'un siphon et d'un entonnement (planche 2), sont protégés par une estacade constituée de madriers et pieux de bois enchevêtrés (planche 3 ).

L'augmentation de la puissance de la centrale prévoit la réalisation des tranches 5 et 6 , de $250 \mathrm{MW}$ chacune, pour un débit de prise de $9 \mathrm{~m}^{3} / \mathrm{s}$. Les deux ouvrages de prise correspondants, de même conception que les ouvrages des prises existantes, seront protégés par une estacade métallique moins compacte (planche 3 ).
1.2 Programme général des essais sur modèle.

Le programme général des essais sur le modèle réduit était le suivant :

- étalonnage hydraulique de la partie de Loire représentée sur le modèle;

- reproduction du phénomène de glaciation et d'aspiration actuel pour différents débits de la Loire et après mise en place des ouvrages de prise des tranches 5 et 6 ainsi que de leur estacade de protection;

- recherche de dispositifs améliorant au maximum la situation actuelle, compte tenu que toute solution de protection ne doit pas empiéter sur le chenal de navigation large de $200 \mathrm{~m}$ au droit des prises d'eau.

Les dispositifs à rechercher doivent être efficaces pour deux sortes de glace:

- les glaces dérivantes en surface (par éléments ou par trains de glace) dont l'épaisseur peut varier dı centimètre au mètre:

- la glace en formation ou « sorbet », composée de microcristaux de glace flottant entre deux eaux, pouvant se trouver soit sous la couche des glaces dérivantes, soit dans les zones d'eau calme et, en particulier, lorsque les glaces dérivantes s'entassent au niveau de l'estacade, en restant prisonnières des pieux. 


\section{R. BONNEFILLE, P. GERMAIN et A. BICHON}

\section{SITUATION DE LA CENTRALE}
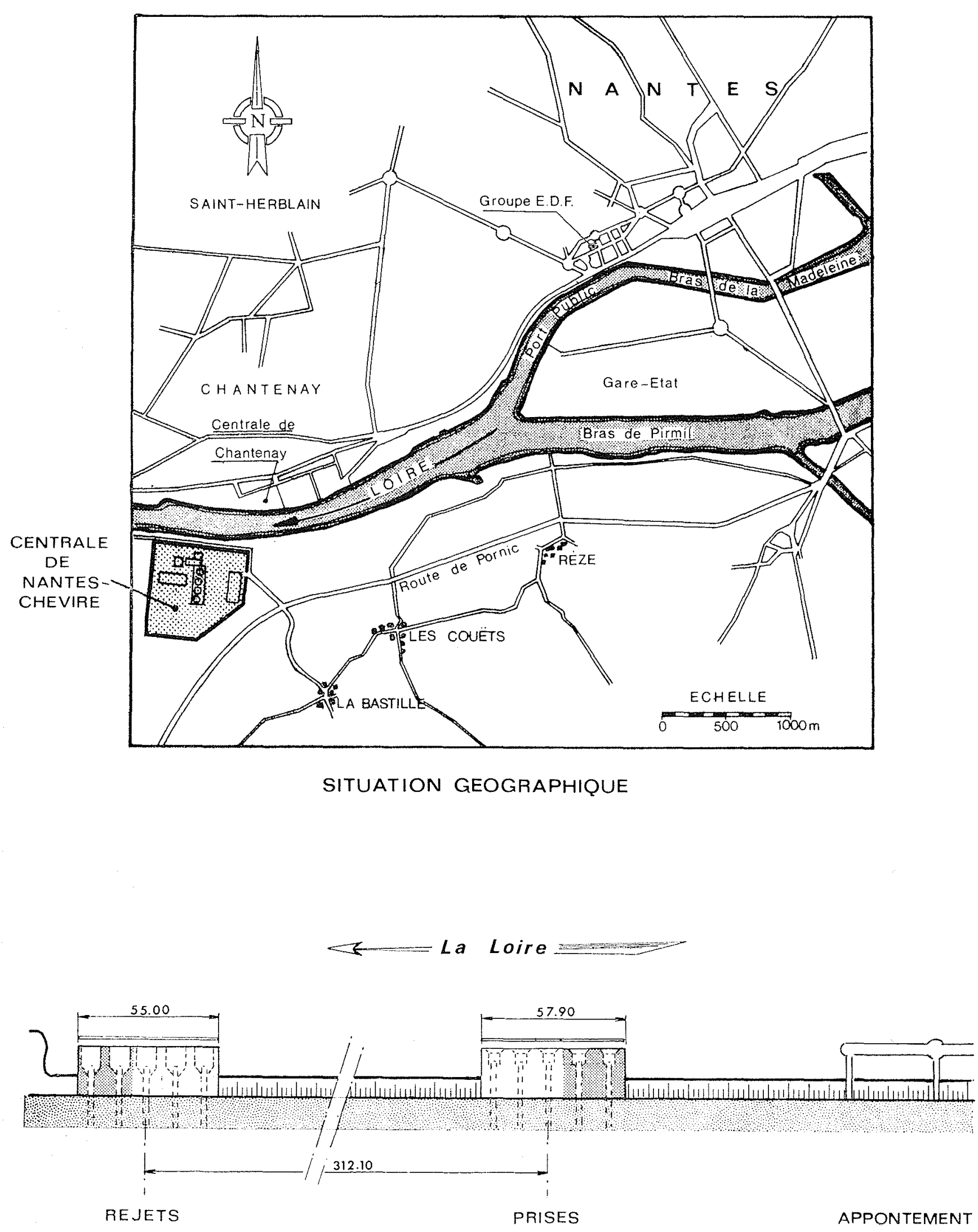

PI. 1 


\section{OUVRAGE DE PRISE}

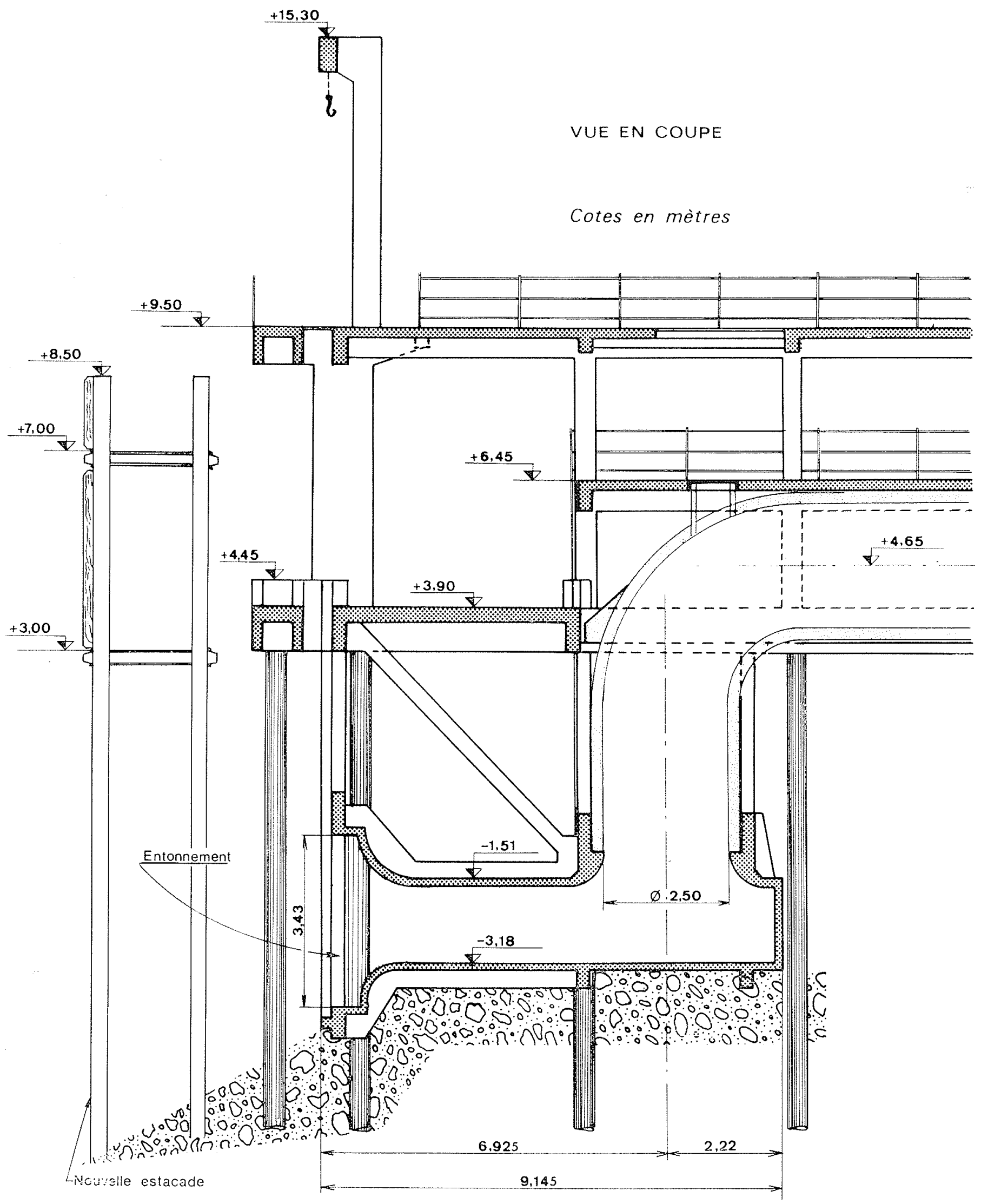

PI. 2 


\section{OUVRAGES DE PRISE}

\&

\section{ESTACADES DE PROTECTION}

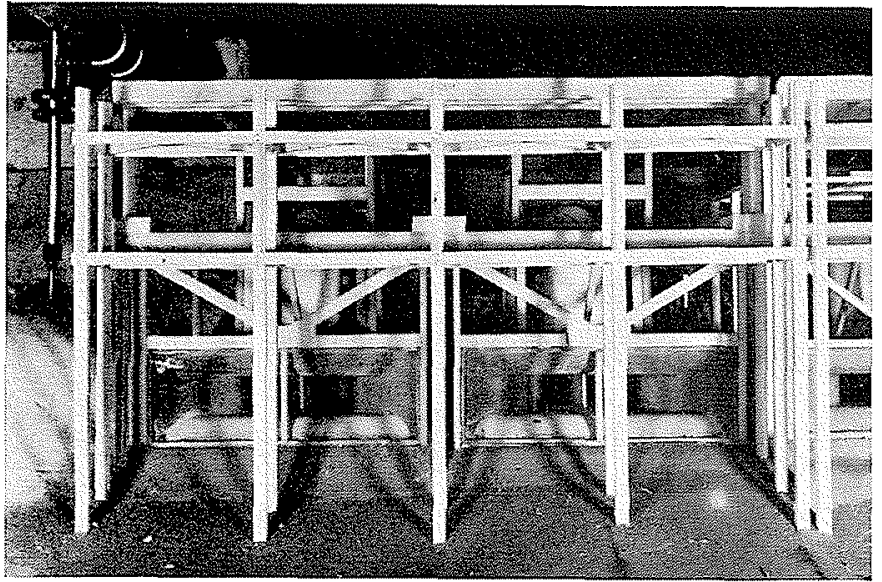

Tranches 5 et 6

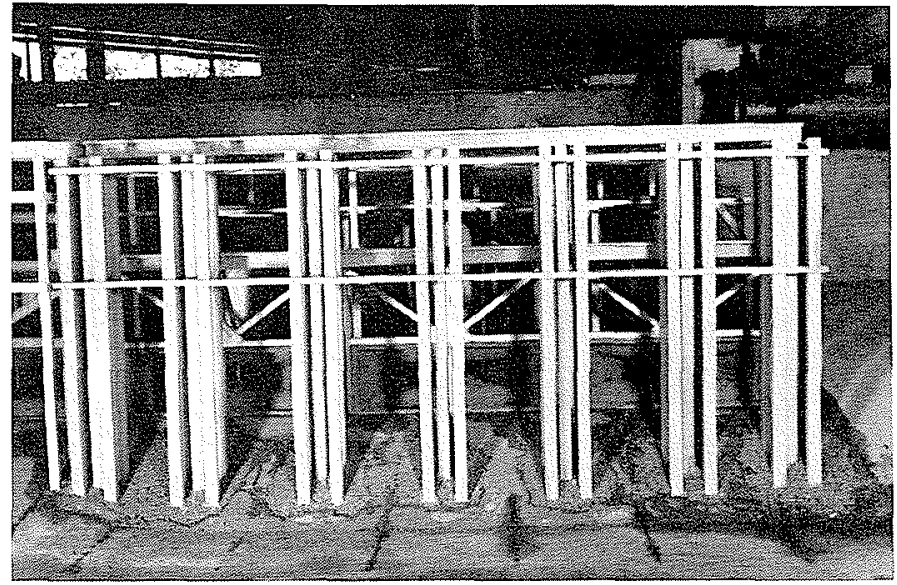

Tranches 1 à 4

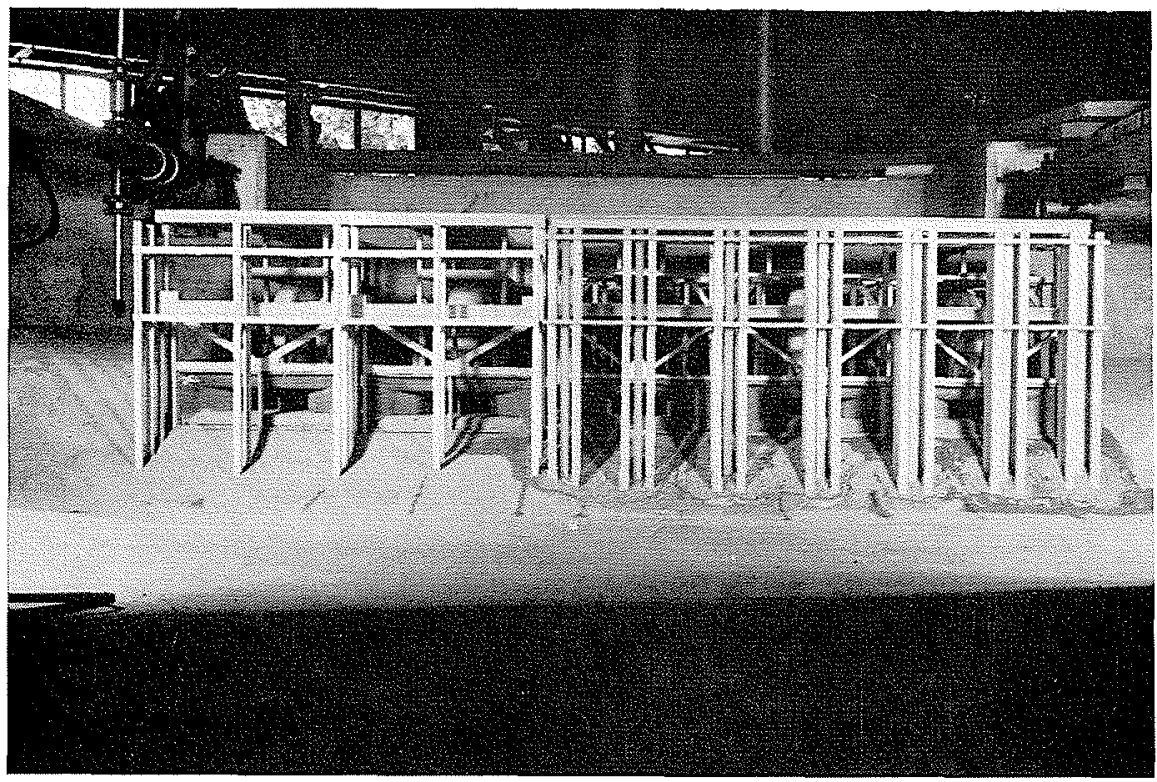

Vue d'ensemble

PI. 3 


\section{Le modèle réduit}

Le modèle est construit à fonds fixes. Le fluide utilisé est de l'eau douce.

\subsection{Condition de similitude : choix de l'échelle.}

L'étude est conduite en similitude de Froude.

L'échelle linéaire, choisie de manière à effectuer des mesures et des observations suffisamment précises, est prise égale à $\lambda=1 / 40$.

Il en résulte, en similitude de Froude, les échelles dérivées suivantes :

- échelle des temps : $\lambda^{1 / 2}=1 / 6,32$

- échelle des vitesses : $\lambda^{1 / 2}=1 / 6,32$

- échelle des débits : $\lambda^{5}=1 / 10119$

\subsection{Description du modèle (planche 4).}

\subsubsection{Représfentation du lit de la LoIRE.}

Le modèle représente le lit de la Loire sur toute sa largeur et sur une longueur d'environ $200 \mathrm{~m}$ de part et d'altre des ouvrages de prise d'eau. Les fonds sont représentés d'après un plan de sondages du Port Autonome de Nantes Saint-Nazaire (sondages de mars et avril 1963 : Feu Haute-Indre - Feu Cheviré). Ces sondages sont complétés par des relevés effectués au droit des estacades de déchargement du fuel et au droit des ouvrages de prise d'eau.

\subsubsection{REPRÉSENTATION DES OUVRAGES.}

Les ouvrages représentés sur le modèle, en afcodur, sont les suivants :

- les trois ouvrages d'aspiration en siphon des quatre premières tranches avec les différentes poutraisons des estacades;

- l'estacade avancée de protection de ces trois ouvrages, d'après des documents photographiques;

- les deux ouvrages d'aspiration en siphon des deux nouvelles tranches avec les différentes poutraisons des estacades;

- l'estacade avancée de protection de ces deux ouvrages;

- l'ancienne estacade de déchargement des navires pétroliers et charbonniers.

\subsubsection{Dispositif D'Alimentation.}

Une fois convenablement rempli, le modèle fonctionne en circuit fermé. Le dispositif d'alimentation comporte :

- deux pompes: l'une pouvant soit être en réserve, soit fonctionner en by-pass, de façon à pouvoir réaliser sur le modèle les trois cas de fonctionnement possibles en période de glace : crue de la Loire, étiage de la Loire, légère renverse à Nantes;

- un système de vannes d'aiguillage et de réglage des débits;

- un appareil de mesure du débit (type OTIC).

\subsubsection{Dispositif DE PRISE.}

Le débit de prise, assuré par une pompe, est réglé par un système de vannes et mesuré par un rotamètre; le système de vannes permet de régler simultanément, ou séparément, le débit de prise des quatre anciennes tranches et des deux nouvelles tranches.

\subsubsection{RÉGLAGE HYDRAULIQUE DU MODÈLE,}

La cote du plan d'eau sur le modèle en fonctionnement, est ajustée soit par une vanne de remplissage située sur le modèle, soit par une vanne de vidange sur le circuit d'alimentation, et mesurée à l'aide de pointes limnimétriques. L'exploration des vitesses d'écoulement est effectuée au moyen d'un moulinet (type OTT).

\subsection{Représentation de la glace.}

\subsubsection{GLACES DÉRIVANTES.}

Les glaçons flottants sont représentés en paraffine $\left(\rho=0,941 \mathrm{~g} / \mathrm{cm}^{3}\right)$ de même densité que la glace naturelle. La paraffine fondue est coulée dans des moules de différentes épaisseurs passés immédiatement au congélateur : on réalise ainsi une trempe facilitant la fabrication des glaçons. Les diverses épaisseurs des moules utilisés correspondent à des glaçons sensiblement cubiques de 4,8 , 16,80 et $120 \mathrm{~cm}$ (nature) d'arête.

\subsubsection{GLACE EN FORMATION.}

Les cristaux composant le «sorbet », en suspension dans l'eau, sont représentés à l'aide d'un mélange convenablement dosé de benzine, de trichloréthylène et de peinture cellulosique blanche. Le mélange ainsi obtenu, de densité voisine de celle de l'eau, est injecté sous pression à l'aide d'aiguilles hypodermiques : les dimensions des petites bulles injectées dépendent de la grosseur de l'aiguille et de la valeur de la pression.

\section{3. - Etude des données}

\subsection{Données naturelles.}

Pour l'étalonnage hydraulique du modèle, les données indispensables en période de prise en glace sur la Loire, sont les suivantes :

- les marées et les enregistrements marégraphiques;

- les débits fluviaux;

- les courants.

En hiver, la Loire est le plus souvent à l'étiage et le courant s'inverse au niveau des prises d'eau. Toutefois, en cas de froid à la fin de l'hiver ou au début du printemps, il peut se produire une crue au moment d'une prise en glace.

La vitesse maximale relevée au niveau de Cheviré, au moment des prises en glace, est de 2,5 nœuds $(\simeq 1,30 \mathrm{~m} / \mathrm{s})$.

\subsubsection{LES MARÉES ET LES ENREGISTREMENTS MARÉgRA- PHIQUES.}

Les enregistrements marégraphiques donnés par les différentes stations régulièrement espacées sur tout le domaine maritime (de Saint-Nazaire à Oudon) permettent à l'aide d'une étude statistique portant sur des moyennes assez nombreuses, d'obtenir les courbes-types de chaque coefficient de marée à chaque station pour un débit fluvial donné.

\subsubsection{LES DÉBITS FLUVIAUX.}

Les débits sont donnés à partir des relevés effectués à Montjean, à $20 \mathrm{~km}$ en aval du confluent de la Loire et de 


\section{LE MODELE REDUIT}

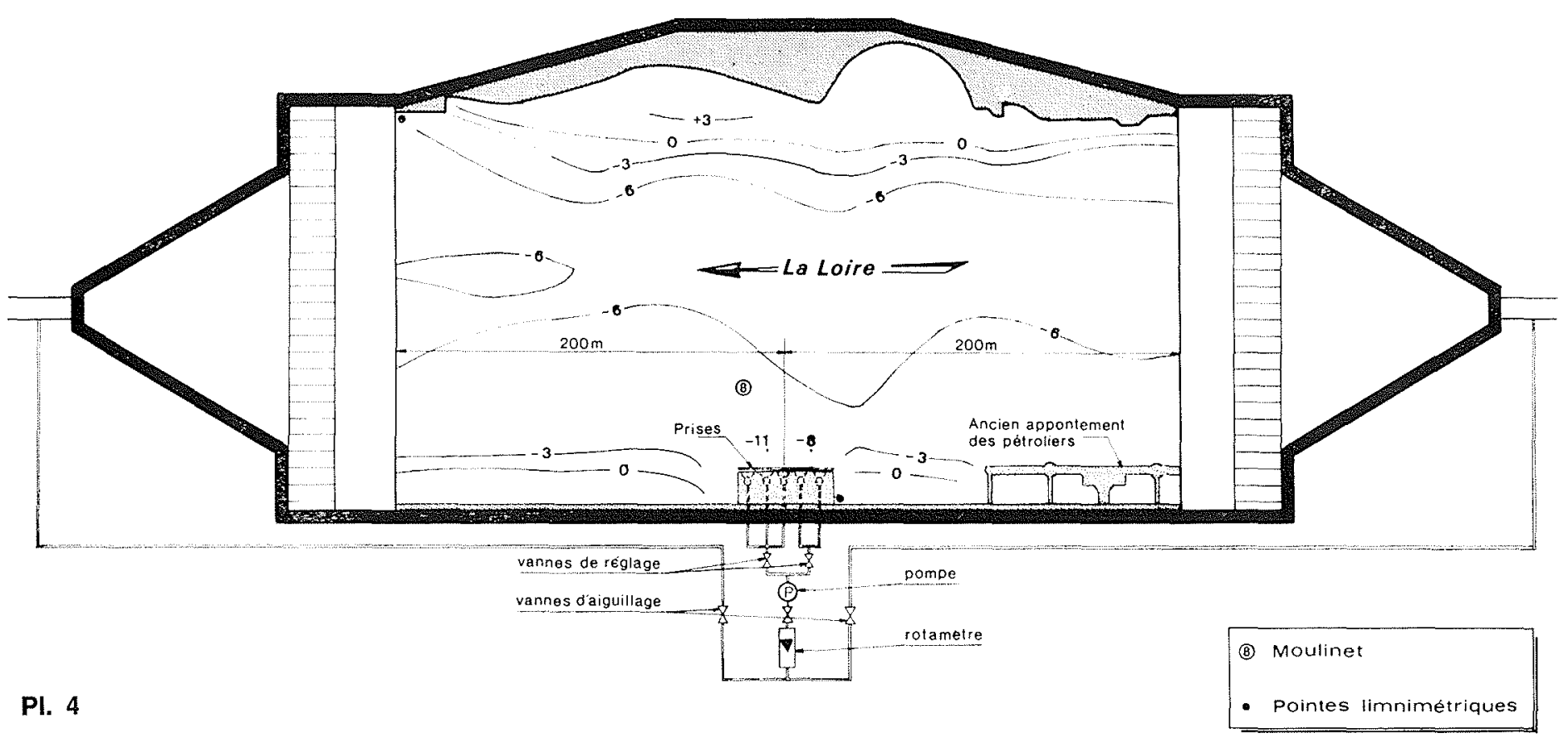

\section{PHENOMENE DE GLACIATION ACTUEL}

19 Février 1963
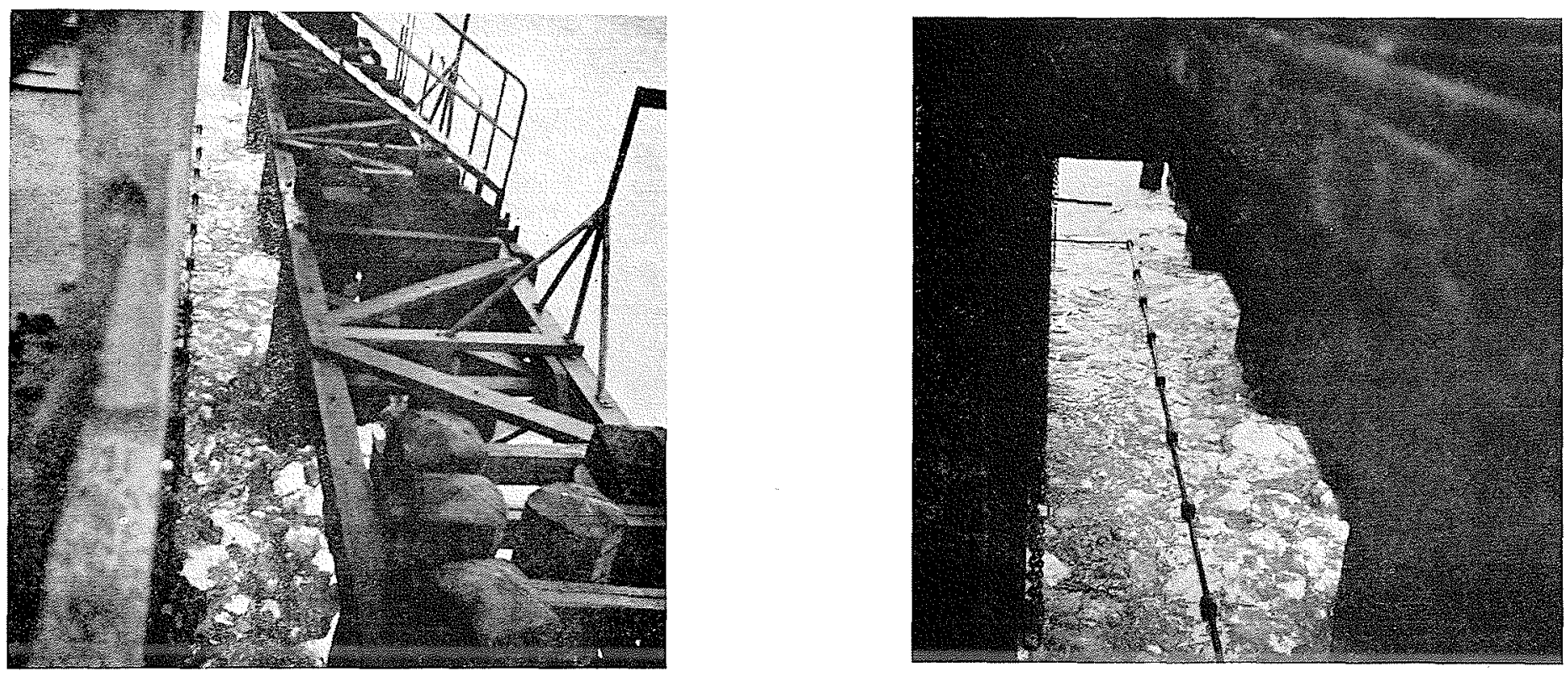

PI. 5 


\begin{tabular}{|c|c|c|c|c|c|c|c|}
\hline \multirow{3}{*}{$\begin{array}{c}\text { COte } \\
\text { A L'ÉCHelle DE } \\
\text { MontJeAn } \\
\\
(\mathrm{m})\end{array}$} & \multirow{3}{*}{$\begin{array}{c}\text { DÉBIT } \\
\text { DE LA LOIRE (*) } \\
\\
\left(\mathrm{m}^{3} / \mathrm{s}\right)\end{array}$} & \multicolumn{6}{|c|}{ Vrtesses maximales (Natuds) a Nantes } \\
\hline & & \multicolumn{2}{|c|}{ Coefficient 45 , morte-eau } & \multicolumn{2}{|c|}{ Coefficient 70 , moyenne-eau } & \multicolumn{2}{|c|}{ Coefficient 94 , vive-eas } \\
\hline & & Flot & Jusant & Flot & Jusant & Flot & Jusant \\
\hline 0 & 220 & 1,1 & 0,8 & 1,7 & 1,1 & 2,2 & 1,4 \\
\hline 1 & 450 & 0,4 & 1,3 & 0,9 & 1,5 & 1,4 & 1,6 \\
\hline 2 & 950 & & 1,7 & 0,2 & 1,8 & 0,5 & 2,0 \\
\hline 3 & 1680 & & 2.2 & & 2,2 & & 2,3 \\
\hline 4 & 2270 & & 2,6 & & 2,7 & & 2,7 \\
\hline 5 & 3050 & & 3,0 & & 3,0 & & 3,1 \\
\hline
\end{tabular}

(*) D’après la courbe expérimentale, hauteur-débit, à Montjean.

la Maine. En période de glace le débit varie généralement de 1000 à $1700 \mathrm{~m}^{3} / \mathrm{s}$ (exceptionnellement le 3 février 1963 il était de $750 \mathrm{~m}^{3} / \mathrm{s}$ à Chantenay tout en étant estimé à $1080 \mathrm{~m}^{3} / \mathrm{s}$ à Montjean). On peut donc prendre comme débits caractéristiques de la Loire au mois de février les débits suivants : $300,750,1300$ et $1700 \mathrm{~m}^{3} / \mathrm{s}$ (ces débits représentant environ les trois quarts des pourcentages cumulés).

\subsubsection{LES COURANTS.}

L'interprétation des nombreuses mesures de courant est délicate, car la vitesse sur une verticale varie en grandeur et en direction avec l'heure de la marée (début ou fin de jusant, période de flot), le coefficient de la marée et le débit de la Loire. Le premier tableau donne les valeurs des vitesses maximales à Nantes, compte tenu du débit de 1a Loire pour trois types de marée.

Remarque : l'absence de nombre dans les cases signifie qu'il n'y a que du jusant.

\subsubsection{PhÉNOMÈne ACTUEL DE GLACIATION.}

Les phénomènes de glaciation actuels ont été observés pour les conditions suivantes :

\begin{tabular}{|c|c|c|}
\hline DATE & $\begin{array}{c}\text { DÉBIT } \\
\text { DE LA LoIRE } \\
\left(\mathrm{m}^{3} / \mathrm{s}\right)\end{array}$ & $\begin{array}{c}\text { CoEFFICIENT } \\
\text { DE LA MARÉE }\end{array}$ \\
\hline 3 février 1963 & 1080 & 53 \\
\hline 19 février 1963 & 1780 & 39 \\
\hline 17 janvier 1966 & 2320 & 52 \\
\hline
\end{tabular}

La planche 5 présente des photographies de la prise en glace le 19 février 1963.

\subsection{Choix des conditions d'essais sur modèle.}

\subsubsection{COTE DU PLAN D'EAU.}

Les hauteurs des basses-mers et des pleines-mers à Nantes pour trois types de marée sont les suivantes :

\begin{tabular}{|c|c|c|c|c|c|c|}
\hline \multirow{3}{*}{$\begin{array}{l}\text { COTE A } \\
\text { L'ÉCHELLE DE } \\
\text { MONTJEAN } \\
\text { (m) }\end{array}$} & \multicolumn{6}{|c|}{$\begin{array}{l}\text { HAUTLUR EN MÈTRES (C.M.) DES basSES-MERS } \\
\text { [TT Pl.EINES-MERS A NANTES }\end{array}$} \\
\hline & \multicolumn{2}{|c|}{$\begin{array}{l}\text { Coefficient } 45 \\
\text { morte-eau }\end{array}$} & \multicolumn{2}{|c|}{$\begin{array}{l}\text { Coefficient } 70 \\
\text { moyenne-eau }\end{array}$} & \multicolumn{2}{|c|}{$\begin{array}{c}\text { Coefficient } 94 \\
\text { vive-eal }\end{array}$} \\
\hline & $\mathrm{BM}$ & $\mathbf{P M}$ & $\mathrm{BM}$ & $\mathrm{PM}$ & $\mathrm{BM}$ & $\mathbf{P M}$ \\
\hline 0 & 1,80 & 4,55 & 1,60 & 5,25 & 1,55 & 5,80 \\
\hline 2,5 & 3,20 & 5,10 & 3,20 & 5.80 & 3,20 & 6,30 \\
\hline 4.5 & 5.40 & 600 & 5,30 & 6,15 & 5,60 & 6,70 \\
\hline
\end{tabular}

La condition d'essai la plus pessimiste étant une bassemer de vive-eau en période d'étiage, la cote du plan d'eau choisi est $+1,50 \mathrm{~m}$ (C.M.).

\subsubsection{DURÉE D'EsSAI.}

Chaque essai doit durer le temps d'une marée basse. En prenant comme durée de la marée basse le temps où la cote $z$ en Loire est, par exemple, inférieure à $+2,00$ m (C.M.), on obtient, en période d'étiage les temps suivants :

\begin{tabular}{|c|c|c|}
\hline Morte-eau & Moyenne-eau & Vive-eau \\
\hline $1,80<z<2,00$ & $1,60<z<2,00$ & $1,55<z<2,00$ \\
\hline $1 \mathrm{~h} 20 \mathrm{mn}$ & $1 \mathrm{~h} 30 \mathrm{mn}$ & $1 \mathrm{~h} 40 \mathrm{mn}$ \\
\hline
\end{tabular}




\section{R. BONNEFILLE, P. GERMAIN ef A. BICHON}

La durée de chaque essai doit donc être de $20 \mathrm{mn}$ modèle (soit 1 h 40 mn nature).

\subsubsection{VITESSES EN LOIRE.}

Les vitesses maximales de jusant à Nantes pour trois types de marée sont les suivantes:

\begin{tabular}{|c|c|c|}
\hline TYPE DE MARÉE & $\begin{array}{l}\text { DÉBIT } \\
\text { DE LA LOIRE } \\
\left(\mathrm{m}^{3} / \mathrm{s}\right)\end{array}$ & $\begin{array}{l}\text { Vitesse MaXIMALE } \\
\text { DE JUSANT } \\
\text { (nœuds) }\end{array}$ \\
\hline $\begin{array}{c}\text { Coefficient } 45 \\
\text { morte-eau }\end{array}$ & $\begin{array}{r}300 \\
750 \\
1300 \\
1700\end{array}$ & $\begin{array}{l}1,1 \\
1,6 \\
1,9 \\
2,1\end{array}$ \\
\hline $\begin{array}{l}\text { Coefficient } 70 \\
\text { moyenne-eau }\end{array}$ & $\begin{array}{r}300 \\
750 \\
1300 \\
1700\end{array}$ & $\begin{array}{l}1,3 \\
1,7 \\
2,0 \\
2,2\end{array}$ \\
\hline $\begin{array}{l}\text { Coefficient } 94 \\
\text { vive-eau }\end{array}$ & $\begin{array}{r}300 \\
750 \\
1300 \\
1700\end{array}$ & $\begin{array}{l}1,5 \\
1,8 \\
2,1 \\
2,3\end{array}$ \\
\hline
\end{tabular}

L'instant où la vitesse de jusant atteint sa valeur maximale est très variable: il dépend du coefficient de la marée et du débit de la Loire. Cependant, en période d'étiage, cette valeur maximale est atteinte deux heures environ après le début du jusant quel que soit le coefficient de la marée.

Les vitesses moyennes d'écoulement en Loire choisies pour les essais sont les suivants : $1,1,5$ et 1,8 nœud.

\section{4. - Reproduction sur modèle du phénomène actuel de glaciation et d'aspiration}

La cote du plan d'eau sur le modèle en fonctionnement fut réglée à - $1,50 \mathrm{~m}$ (C.M.); la durée de chaque essai correspondait à $1 \mathrm{~h} 40 \mathrm{mn}$ (nature); les vitesses moyennes d'écoulement en Loire étaient 1, 1,5 ou 1,8 nœud.

Les glaces dérivantes, utilisées pour les essais, correspondaient aux dimensions moyennes suivantes: $0,40,0,80$ ou 1,20 m (nature); l'alimentation des glaces sur le modèle était effectuée, pendant toute la durée de l'essai, au voisinage de l'ancienne estacade de déchargement des navires pétroliers et charbonniers.

Les trois ouvrages de prise des tranches 1 à 4 étaient représentés avec leur estacade de protection, type ancienne (madriers et pieux de bois enchevêtrés) et les deux ouvrages de prise des tranches 5 et 6 avec leur estacade de protection, type nouvelle (métallique et moins dense).

La planche 6 présente à titre d'exemple les résultats d'un essai.

Quelles que soient la vitesse moyenne d'écoulement en Loire et les dimensions des glaces dérivantes:

- les madriers et pieux de l'ancienne estacade, très enchevêtrés, favorisent la prise en glace des ouvrages des tranches 1 à 4 : les glaces dérivantes s'entassent au niveau de l'estacade et restent prisonnières des pieux;

- un large mouvement tourbillonnaire des glaces dérivantes prend naissance à travers les ouvrages de prise des tranches 5 et 6 , du fait du décollement des lignes de courant provoqué par la pointe avancée du talus immédiatement à l'amont des ouvrages de prise.

\section{5. - Dispositifs de protection contre les glaces dérivantes}

\subsection{Remplacement de l'ancienne estacade de protection des} ouvrages de prise des tranches 1 à 4 .

L'ancienne estacade de protection des ouvrages de prise des tranches 1 à 4 est remplacée par une estacade de même longueur, et de conception identique à celle, métallique, devant protéger les ouvrages de prise des tranches 5 et 6 (planche 7).

Les essais furent effectués dans les mêmes conditions que précédemment (planche 8 donnée à titre d'exemple).

En conclusion, le remplacement de l'ancienne estacade par une estacade semblable à celle devant protéger les ouvrages de prise des tranches 5 et 6 , apporte une amélioration considérable. Il n'y a pratiquement plus de concentration de glaces dérivantes dans les ouvrages de prise; par contre, le mouvement tourbillonnaire à travers les ouvrages de prise des tranches 5 et 6 est maintenu.

\subsection{Mise en place d'un masque.}

Un masque, construit en palplanches, fut disposé le long de l'estacade côté Loire. Ce masque était réglable en hauteur sur le modèle.

Les essais furent effectués pour différents calages de la partie inférieure du masque : à $+1,20,+0,80$ et $+0,50 \mathrm{~m}$ (C.M.); les vitesses moyennes d'écoulement en Loire variant de 1 à 1,8 noeud; la cote minimale actuelle de la Loire à Nantes-Chantenay était de $+1,50 \mathrm{~m}$ (C.M.) avec possibilité dans l'avenir de descendre à $+1,20 \mathrm{~m}$ (C.M.).

En conclusion, le masque peut être, dans un premier stade, calé à la cote $+0,80 \mathrm{~m}$ (C.M.). Dans ces conditions, la vitesse moyenne d'entrée dans les entonnements des prises d'eau (variant actuellement de 0,21 à $0,26 \mathrm{~m} / \mathrm{s}$ suivant les tranches) serait réduite à $0,10 \mathrm{~m} / \mathrm{s}$ environ sous le masque (planche 9).

\subsection{Prolongement du masque par deux guideaux.}

Le guidage des glaces dérivantes par le masque doit être complété par deux guideaux. Ces guideaux, réalisables en palplanches, seraient de véritables rideaux battus. Le rideau amont serait oblique s'appuyant soit sur un début de rideau existant, soit sur la pointe avancée du talus immédiatement à l'amont des ouvrages de prise. Le rideau aval pourrait, sans grand risque d'accumulation de glaces dérivantes, être implanté au plus court.

Les essais furent effectués pour différentes cotes du plan d'eat en Loire (allant de la basse mer à la pleine mer de vive eaul) et pour différentes vitesses moyennes d'écoulement en Loire.

La planche 10 donne les résultats obtenus:

- croquis de l'implantation générale des ouvrages avec la position optimale du rideau amont (obtenue sur le modèle pour différentes cotes du plan d'eau en Loire); 


\section{REPRODUCTION SUR MODELE DU PHENOMENE DE GLACIATION}

\section{ET D'ASPIRATION ACTUEL}
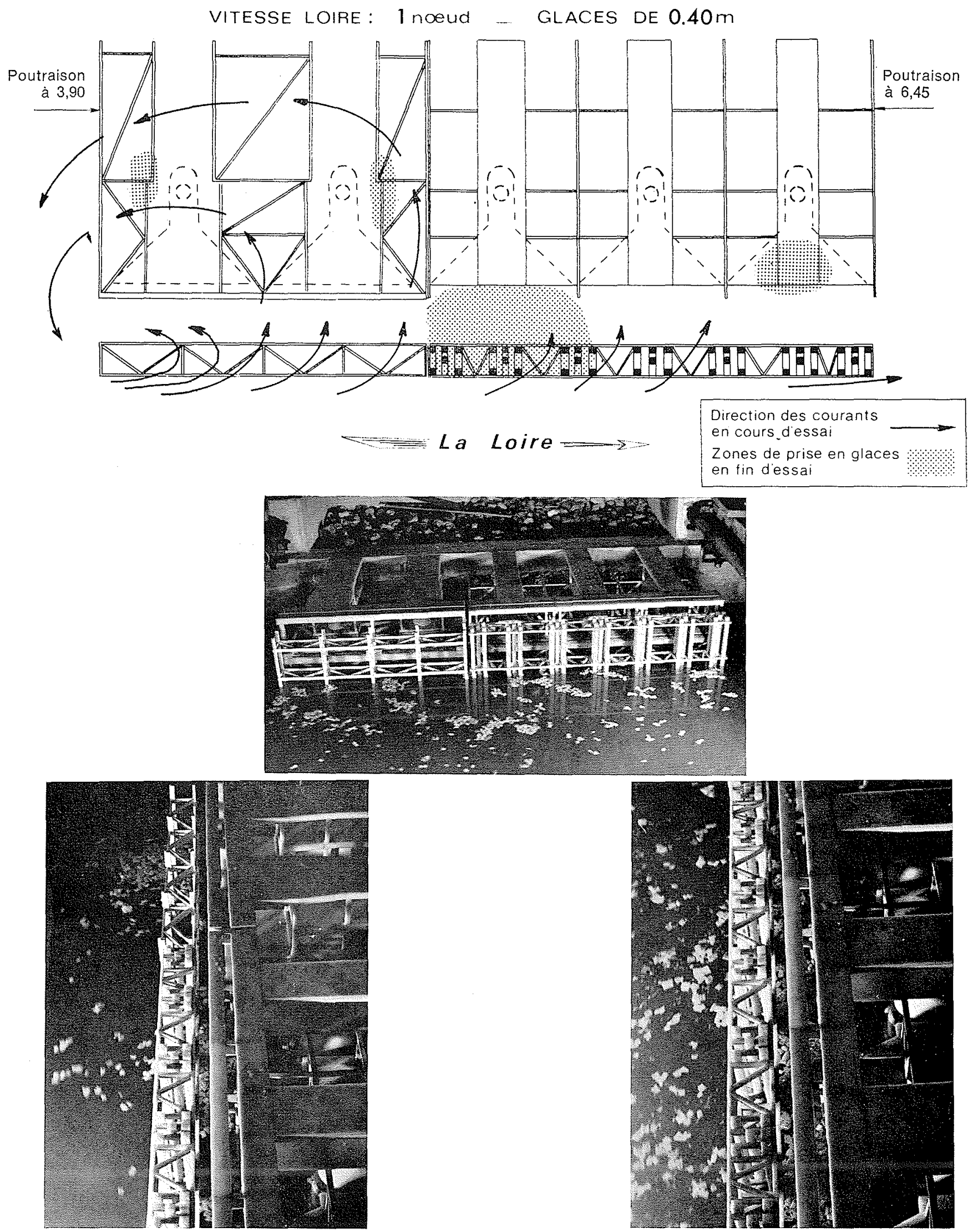


\section{OUVRAGES DE PRISE \\ \& \\ ESTACADES DE PROTECTION}

TRANCHES 1 A 4

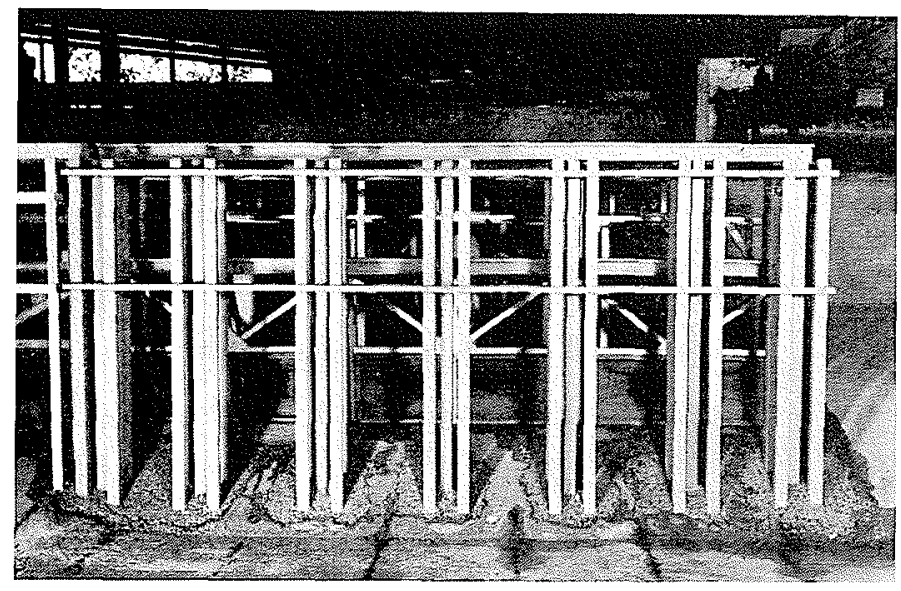

Avec ancienne estacade

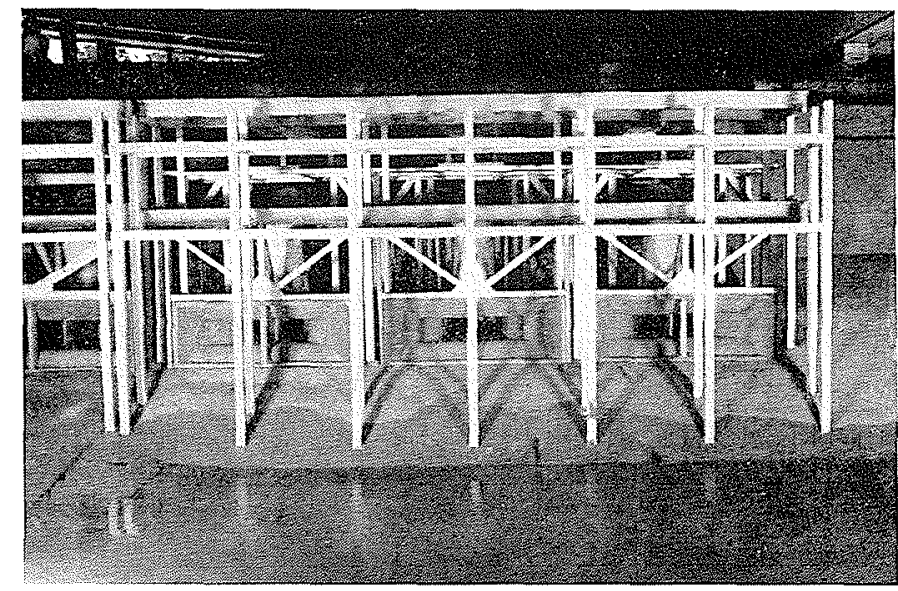

Avec nouvelle estacade

VUE D'ENSEMBLE

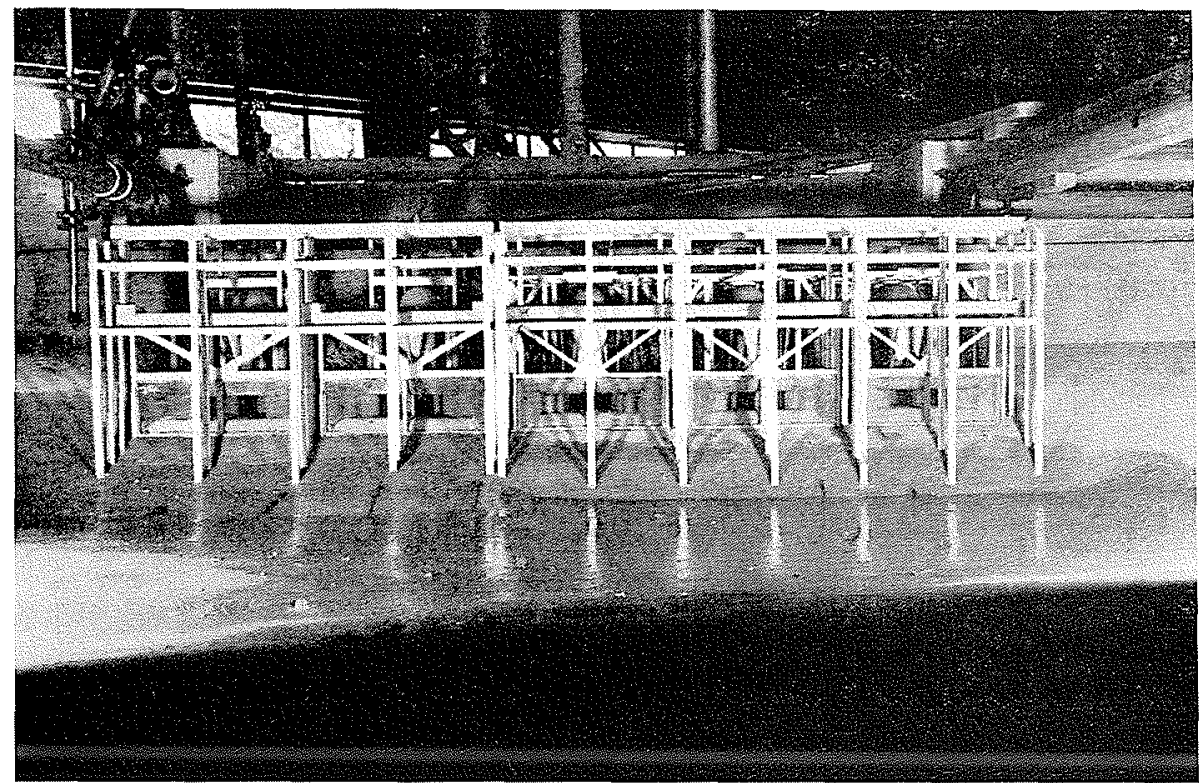

PI. 7

Après remplacement de l'ancienne estacade par la nouvelle 
REMPLACEMENT DE L'ANCIENNE ESTACADE DE PROTECTION DES OUVRAGES DE PRISE DES TRANCHES 1 A 4
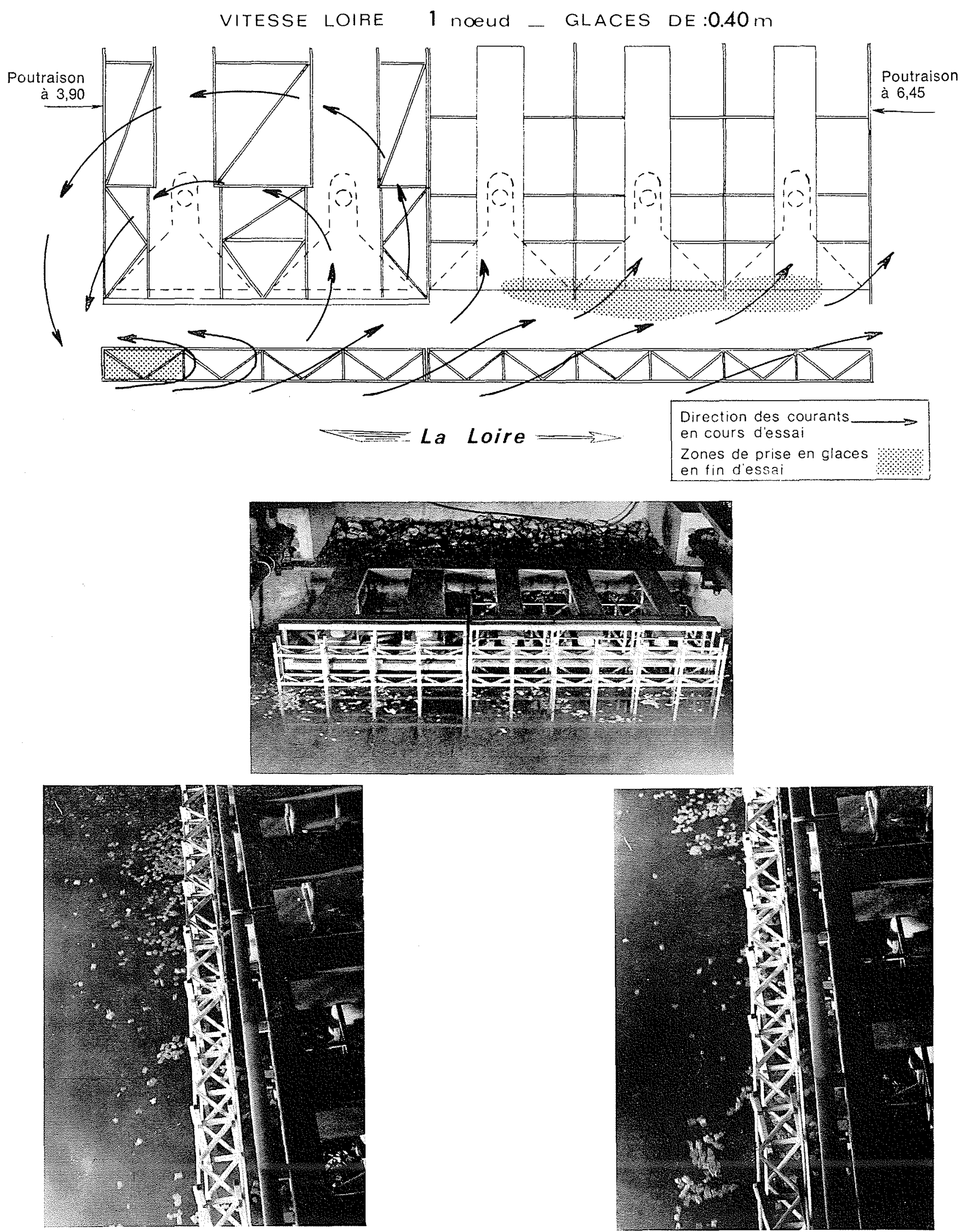


\section{MISE EN PLACE D'UN MASQUE}

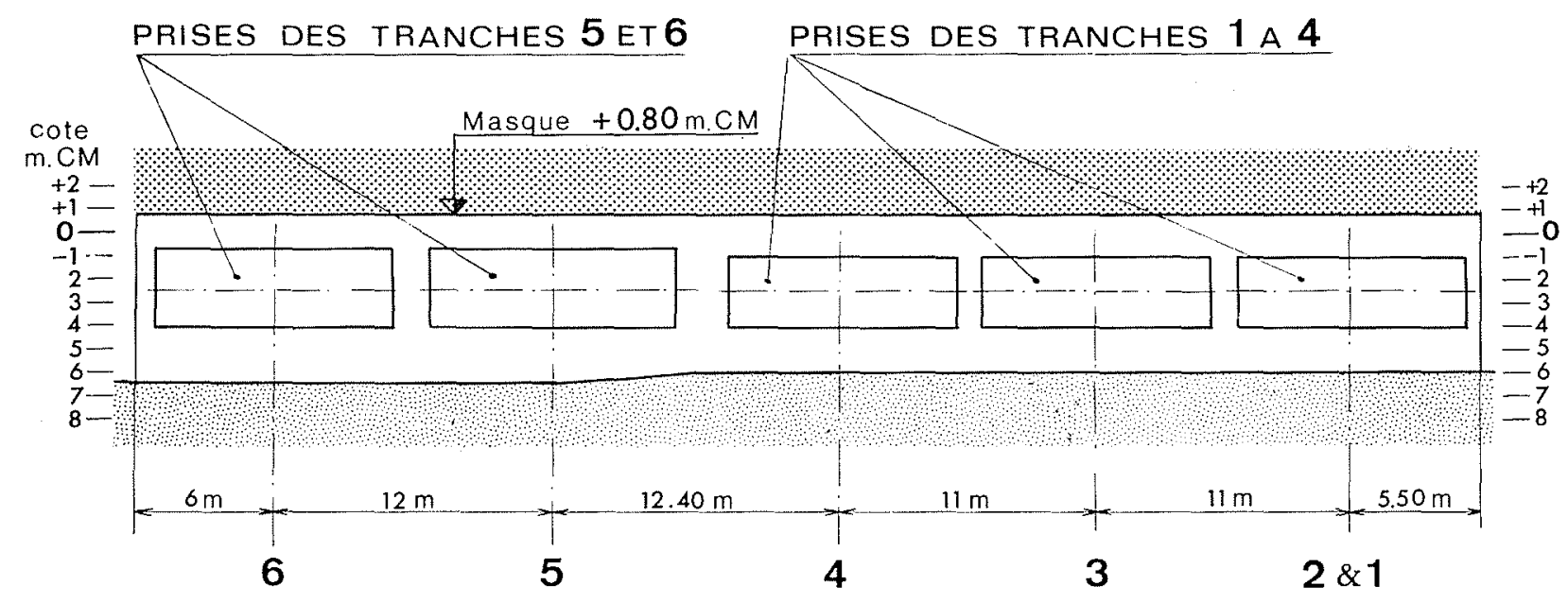

\begin{tabular}{|c|c|c|c|c|c|}
\hline TRANCHES & 6 & 5 & 4 & 3 & $2 \& 1$ \\
\hline $\begin{array}{l}\text { Section d'entree a } \\
\text { l'ouvrage de prise } \mathrm{m}^{2}\end{array}$ & 35 & 35 & 29.1 & 29.1 & 29.1 \\
\hline Debit de prise $\mathrm{m}^{3} / \mathrm{s}$ & 9 & 9 & 7 & 6 & 6 \\
\hline $\begin{array}{l}\text { Vitesse moyenne } \\
\text { d'entrée } \mathrm{m} / \mathrm{s}\end{array}$ & 0.26 & 0.26 & 0.24 & 0.21 & 0.21 \\
\hline Sous le masque & \multicolumn{2}{|c|}{$\mathrm{S}=390 \mathrm{~m}^{2}$} & $\mathrm{Q}=37 \mathrm{~m} / \mathrm{s}$ & \multicolumn{2}{|c|}{$v_{m}=0.095 \mathrm{~m} / \mathrm{s}$} \\
\hline
\end{tabular}

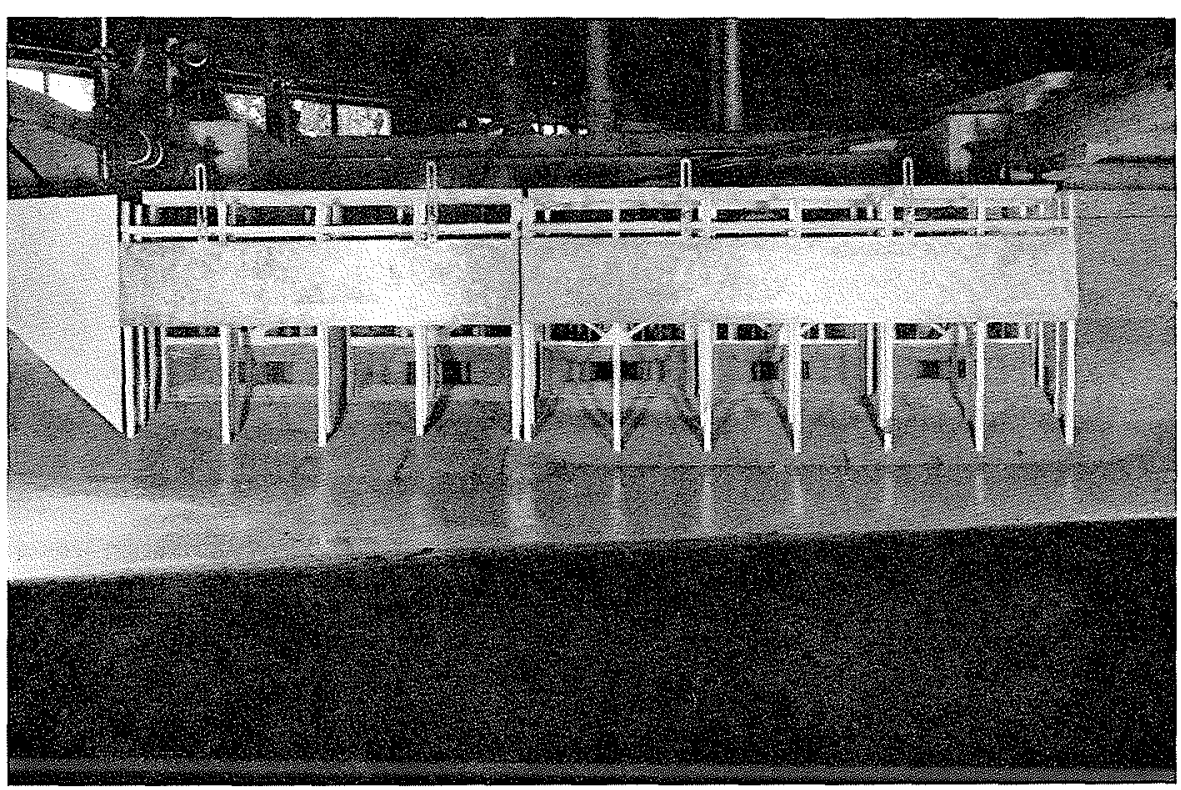

PI. 9 


\section{MASQUE ET GUIDEAUX}

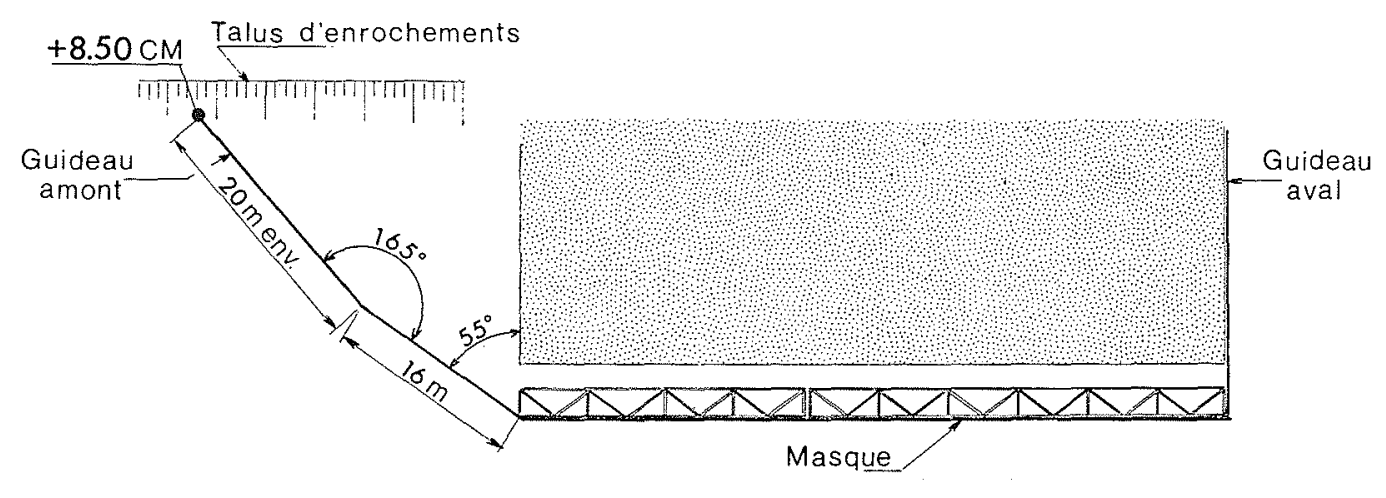

$\longrightarrow$ La Loire $\longrightarrow$
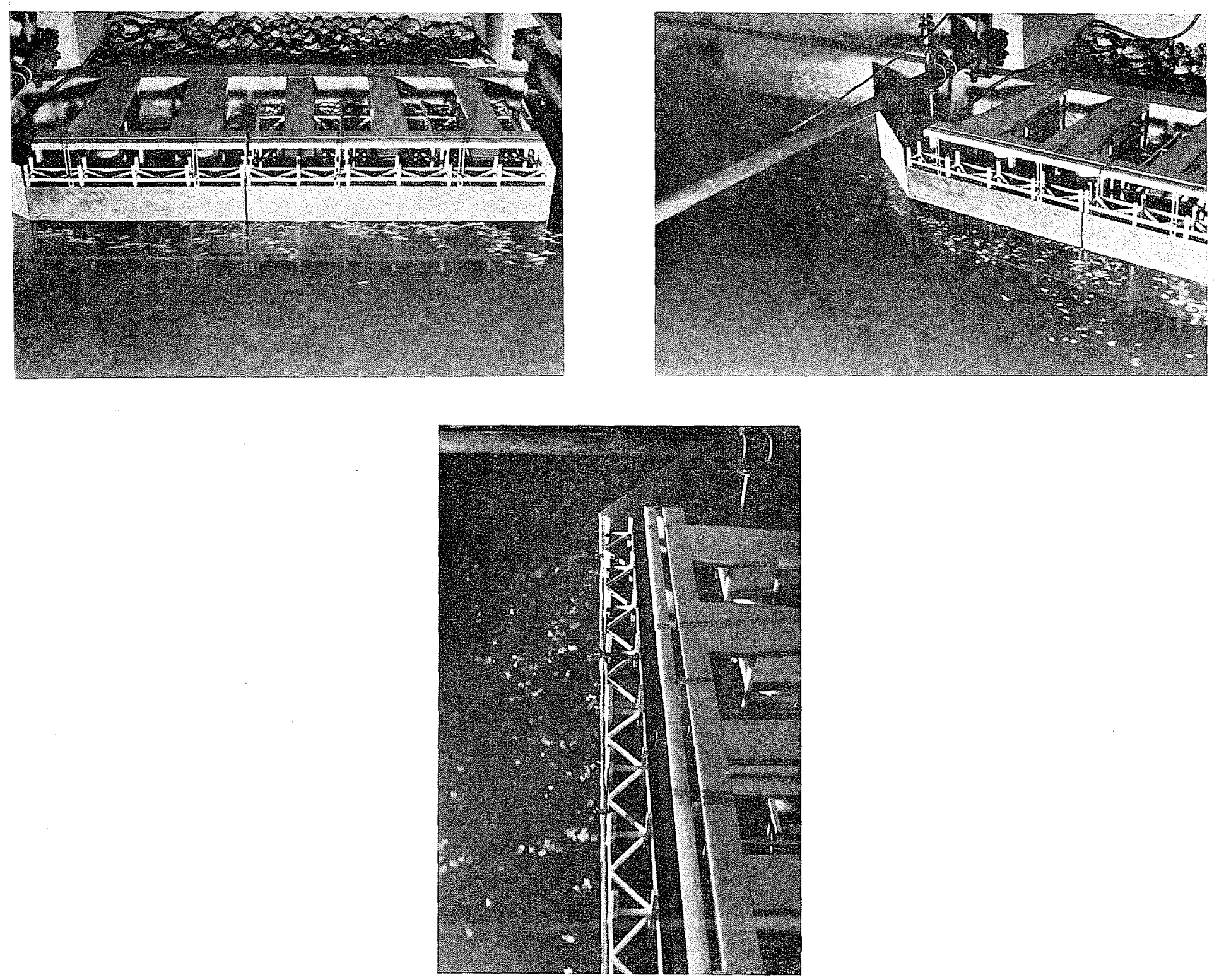

PI. 10 


\section{R. BONNEFILLE, P. GERMAIN et A. BICHON}

- photographies de l'écoulement montrant l'efficacité totale du guidage des glaces dérivantes par le masque et le rideau amont

\section{Remarque.}

Un dispositif de vidange des eaux de surface de l'enceinte créée par le masque et les deux guideaux, devrait être prévu afin d'éviter la stagnation de l'eau en été et une prise en glace facile en hiver.

\section{6. - Lutte contre la formation du "sorbet": protection par barrage d'air comprimé}

\subsection{Principe du barrage d'air comprimé.}

En immergeant au fond d'une nappe d'eau une tubulure munie de gicleurs et alimentée par une source d'air comprimé, on établit, au-dessus de chaque gicleur, un courant continu de bulles d'air ascendantes. Il se produit à la surface de l'eau au-dessus de chaque gicleur un monticule à partir duquel l'eau s'écoule en tous sens; l'eau s'écoulant est renouvelée par le courant ascendant provoqué par la vitesse des bulles d'air. Le volume du monticule est égal à la somme des volumes des bulles d'air contenues dans l'eau entre le gicleur et la surface.

Les monticules forment un bourrelet continu et l'eau s'écoule de part et d'autre à une vitesse dépendant des grandeurs suivantes :

- diamètre et longueur de la tubulure;

- ajutage et écartement des gicleurs;

- pression et débit de l'air comprimé;

- profondeur d'eau.
Il est, par ailleurs, indispensable que tous les gicleurs soient alimentés uniformément d'un bout à l'autre du barrage.

6.2 Etant donné la complexité du phénomène à représenter, des essais seulement qualitatifs ont été effectués à l'échelle $1 / 30$.

Le brassage obtenu par le rideau de bulles d'air est efficace : le courant continu des bulles d'air entraine dans son mouvement ascendant le «sorbet $»$ et crée une agitation de la surface du plan d'eau derrière le masque, diminuant ainsi les risques de formation de glaces dans l'enceinte formée par le masque et les deux guideaux. Ainsi, le rideau de bulles d'air peut éviter ou retarder la formation du «sorbet $»$ et son entrée dans les prises d'eau.

Bibliographie sommaire

Birr (P.-J.). - « Prévention de la formation de la glace sur les ouvrages hydrauliques». Water Power, p. 136-141 (avril 1954) et p. 185-189 (mai 1954).

A.I.R.H.: Huitième Congrès. - Séminaire $S_{1}$ 《 Le problème des glaces dans les structures hydrauliques $\gg .29$ communications (Montréal 1959).

Simmonds (A.-T.). - « Prevention of ice formations by air bubbling 》. Proceedings of the Eastem Snow Conference, vol. 2, p. 37-39 (1953-1954). A.I.H.S., p. 390-398 (1948).

CARSTENS (T.). - «Experiments with supercooling and ice formation in flowing water ». Geofysiske Publikasioner Geophysica Norvegica, vol. XXVI, $\mathrm{n}^{0} 9$ (décembre 1966).

Cartens (T.). - Hydraulics of river ice. La Houille Blanche, $\mathrm{n}^{\circ} 4$, p. 271-284 (1968)

Mater (C.-G.). - « Producing small bubbles of gas in liquids by submerged orifices ». U.S. Bureau of Mines, Bull. $\mathrm{n}^{\circ} 260$, p. 62-120 (1927)

Otogoro, MiYagi. - « The motion of air bubbles rising in water ». philosophical Magazine, London, v. 50, série 6 (juillet 1925).

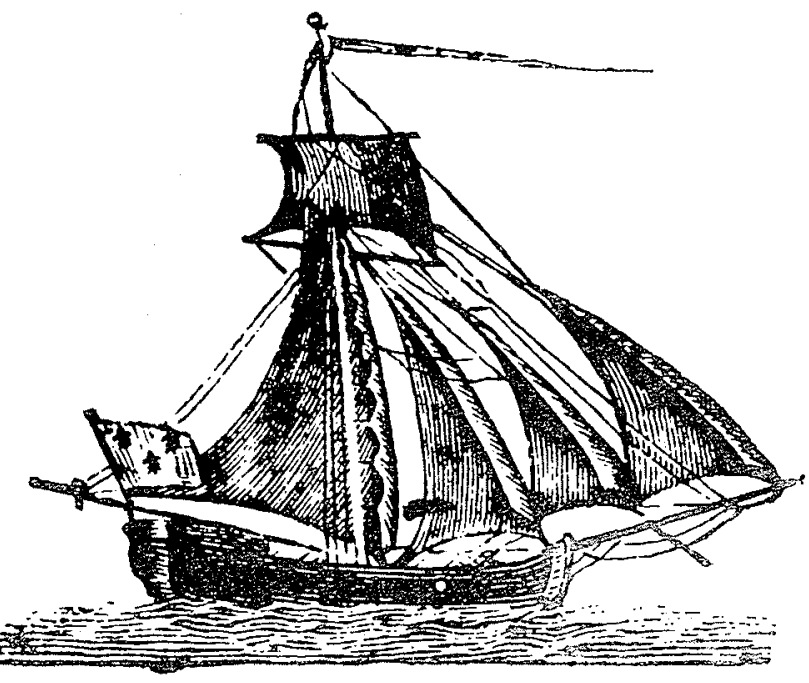

